Courrier du Centre international Blaise Pascal

16 | 1994

Varia

\title{
Le concept d'« homme » dans les Pensées
}

\section{Christian Meurillon}

\section{OpenEdition \\ Journals}

Édition électronique

URL : http://journals.openedition.org/ccibp/589

DOI : $10.4000 /$ ccibp.589

ISSN : 2493-7460

\section{Éditeur}

Centre international Blaise Pascal

\section{Édition imprimée}

Date de publication : 5 avril 1994

Pagination : 13-20

ISSN : 0249-6674

\section{Référence électronique}

Christian Meurillon, "Le concept d'« homme » dans les Pensées», Courrier du Centre international Blaise Pascal [En ligne], 16 | 1994, mis en ligne le 07 janvier 2016, consulté le 02 mai 2019. URL : http:// journals.openedition.org/ccibp/589; DOI : 10.4000/ccibp.589

Ce document a été généré automatiquement le 2 mai 2019.

Centre international Blaise Pascal 


\title{
Le concept d'« homme » dans les Pensées
}

\author{
Christian Meurillon
}

1 Las ! Quel sujet ! Sur un « mot primitif »... L'Esprit géométrique nous dissuade pourtant d'en traiter de semblables. Plus fâcheux, ce sujet, la définition d'homme, c'est celui-là même que Pascal livre en pâture à son ironie. "Quelle nécessité y a-t-il d'expliquer ce qu'on entend par le mot homme? Ne sait-on pas assez quelle est la chose qu'on veut désigner par ce terme? Et quel avantage pensait nous procurer Platon, en disant que c'était un animal à deux jambes sans plumes? Comme si l'idée que j'en ai naturellement, et que je ne puis exprimer, n'était pas plus nette et plus sûre que celle qu'il me donne par son explication inutile et même ridicule; puisqu'un homme ne perd pas l'humanité en perdant les deux jambes, et qu'un chapon ne l'acquiert pas en perdant ses plumes ${ }^{1}$. »

2 Faut-il s'aventurer sur une voie où le maitre de l'Académie s'est ridiculisé ? Mieux vaut ne pas risquer de remplacer les jambes et les plumes de sa définition par des désirs de lièvre et des fascinations de brocatelle, ni, dans une moindre ambition et selon un plus modeste modèle, jouer les P. Noël de l'anthropologie en proposant des définitions circulaires comme il le faisait pour la lumière. Entre obscurcissement et tautologie semble ne s'ouvrir aucun chemin.

3 Cependant, ce que Pascal récuse ici à propos des mots primitifs, ce sont leurs définitions, mais évidemment pas leur emploi. Il est permis de les insérer dans des propositions qui les dotent d'un prédicat dont la pertinence est sujette à vérification et à contradiction. En effet les définitions «ne sont faites que pour désigner les choses que l'on nomme » et donc pour établir explicitement « le rapport entre le nom et la chose », rapport commun, dans le cas des mots primitifs, à tous ceux qui parlent une même langue. Elles ne visent nullement à «montrer la nature » de cette chose ${ }^{2}$. N'est donc ni interdite ni inutile une "science de l'homme", dont l'apologie a du reste un besoin impérieux, à la fois pour fonder une stratégie en vue de la persuasion et pour unifier son discours sur l'état de l'homme. 
4 Cette étude visera essentiellement le concept proprement dit, et non le référent, qui donnerait lieu à une présentation synthétique de l'anthropologie pascalienne. Ne seront donc pas systématiquement énumérées les parties et les propriétés de la chose mais les traits spécifiques du signe linguistique qui identifie le concept parmi tous les autres et assure sa permanence à travers le temps, et particulièrement à travers l'œuvre considérée. Or ces traits ne se ramènent pas aux représentations communes suscitées dans l'esprit de chacun par le mot homme, mais résultent de l'emploi que Pascal fait de ce terme et du réseau de rapports de substitution, de similitude et d'opposition dans lequel il l'intègre et par lequel il le spécifie.

5 L'analyse des données quantitatives permet une appréhension d'ensemble de l'emploi du terme dans les Pensées et en souligne immédiatement quelques caractéristiques majeures.

6 La fréquence d'abord est significative. On sait que Dieu est le substantif le plus employé dans les Pensées. On ne sera pas surpris de constater qu'homme(s) occupe la deuxième place (avec un total de 531 occurrences contre 634 pour Dieu), loin devant chose(s) en troisième position ${ }^{3}$. Nette confirmation quantitative de ce qu'on savait par ailleurs : que l'apologie, qui veut tourner l'homme vers Dieu, ne reconnait vraiment que ces deux centres d'intérêt et les met en valeur parmi tous les autres.

On peut en revanche passer plus rapidement sur la distribution de ces occurrences entre liasses classées et autres unités des Pensées. Elles s'y répartissent en effet par moitiés. On notera simplement que les occurrences barrées sont beaucoup plus nombreuses dans les premières que dans les secondes ${ }^{4}$ : cette surreprésentation s'explique par leur présence répétée dans les grands développements en cours d'élaboration où abondent les repentirs. Il est également intéressant de vérifier que, fort logiquement, la partie des liasses classées consacrée à l'analyse de la condition de l'homme selon la seule lumière naturelle comporte beaucoup plus fréquemment le terme d'homme que la partie du même classement qui prend explicitement en compte la révélation chrétienne ${ }^{5}$.

8 Les conclusions que l'on peut tirer d'un examen quantitatif ne se bornent pas à ces quelques remarques. Il suffit de faire intervenir un critère nouveau : ce sera l'opposition entre deux sortes de détermination, par l'article défini ou par l'article indéfini. Les divergences s'avèrent dès lors de grande portée.

9 Parmi les différents emplois de l'article défini, d'abord, Pascal opère un choix brutal. Les Pensées ne recourent en effet jamais à sa fonction d'anaphorique: jamais l'homme ne représente un homme qui serait antérieurement présent dans le texte. En revanche, la détermination générique, celle qui affirme "l'espèce dans toute son étendue ${ }^{6}$ ", caractérise vivement l'écriture pascalienne qui traite «l'homme» comme un leitmotiv, aussi bien d'un fragment à l'autre qu'à l'intérieur du mouvement d'un même fragment. De plus, l'homme apparaît fréquemment comme complément de nom dans une série de locutions comme «description de l'homme» (éd. Sellier, fr. 113) ou «la faiblesse de l'homme » $(68,130,648)$; mais, corrélativement, beaucoup plus rarement comme sujet d'un verbe (par exemple : « l'homme est vain », 127).

10 Ces emplois signalent nettement la propension de Pascal à l'abstraction: son discours s'attache à la fois à affirmer le concept d'homme comme tel, en subsumant tous les individus sous un même genre, et à envisager ce concept dans sa plus grande extension. Il est clair également que cette présence forte impose au lecteur l'idée que la nature de la réalité représentée est indépendante de sa nomination. La construction du concept par l'esprit apparaît comme seconde : elle ne vise qu'à signifier de façon adéquate la nature 
préexistante de la chose. Quelle que soit en l'occurrence l'essence contradictoire et fuyante de l'homme, la pensée de Pascal apparaît bien essentialiste.

11 Ce mouvement vers la généralisation semble cependant parfois abusif et il n'est pas rare de surprendre Pascal à affirmer des hommes en général dans tel fragment ce que tel autre montre ne valoir que pour une partie d'entre eux, même très majoritaire. Ainsi lit-on que «les hommes ont mépris pour la religion ${ }^{7}$ " (46). II est pourtant des dévots et des apologistes! Erreur? Négligence de pensée? Certainement pas. Mais simplement généralisation hyperbolique, qui fait affirmer du genre ce qui ne convient qu'à une espèce. Dans l'exemple précédent ils peut s'analyser comme un mixte de tous (je fais partie des hommes comme objet) et de les autres (dont je suis distinct comme sujet connaissant, voire comme habile qui se comporte différemment) : la communauté de condition en effet n'implique pas une similitude de morale.

La volonté tactique de dramatiser une situation ne suffit cependant pas à rendre compte de cet apparent excès de généralisation. La vraie raison s'en découvre aisément sur un autre plan, celui de la pensée de derrière. Celle-ci est en effet parfaitement fondée à saisir les hommes comme un ensemble dans leur corruption essentielle, même s'il convient, sur le plan des comportements et des choix de vie, d'opérer des distinctions. Comme l'a enseigné la religion chrétienne, «l'homme naît en péché » $(680$, p. 473). Tous les hommes partagent le même héritage ${ }^{8}$.

13 Notons enfin, dans l'emploi de l'article défini, un cas intéressant d'opposition entre singulier et pluriel, qui caractérise le discours de la Sagesse de Dieu (182). Celle-ci use en effet du singulier pour l'état d'innocence et du pluriel pour celui de corruption. Cette différence grammaticale apparaît bien significative des vérités ici révélées. Qu'est la Chute en effet, sinon le passage pour l'homme de l'unité de son objet d'amour qu'était Dieu à l'inépuisable diversité des biens qu'il tente de lui substituer? Avec elle naît une humanité plurielle et émiettée.

14 L'indéfini un homme, quant à lui, est beaucoup moins souvent employé dans les Pensées. Pour minoritaire qu'il soit, il n'en joue cependant pas moins un rôle important, dans lequel on peut distinguer deux fonctions. D'un côté il rend imaginable (en concrétisant), de l'autre il exemplifie et, en ce sens, finit lui aussi par généraliser : ce qui est dit d'un homme est extensible à n'importe quel homme. C'est ce que montrent bien les saynètes qui animent de leur pittoresque visuel les analyses générales de la condition humaine : " un homme vit avec plaisir en son ménage " (114), "un homme vêtu de brocatelle » (123), « un homme dans un cachot» (195). Une leçon s'impose, toujours semblable : le général doit se voir dans le particulier et, inversement, ce dernier a pour fonction de rendre visible un homme élevé au rang de type.

Une autre voie que celle de la détermination permet d'étudier la notion d'homme, celle de l'examen des substitutions que suscite le déroulement des textes. celles-ci révèlent en effet les équivalents à homme qui surgissent sous la plume de Pascal sans qu'il ait forcément élaboré un système volontaire d'homonymie qui soit valable en dehors du contexte considéré. Mais, par principe, de tels remplacements installent la notion d' homme dans une série de rapports qui aident à l'identifier. Pronoms personnels aussi bien que substantifs peuvent reprendre homme, mais les premiers le font d'une façon plus inattendue que les seconds.

16 Deux cas principaux se présentent pour les pronoms personnels : la troisième personne bien sûr, mais aussi la première personne du pluriel. En effet Pascal ne se contente pas de 
l'anaphorique il(s). Mais, dans la perspective mobile qui est la sienne, il change assez fréquemment de personne verbale et n'hésite pas à glisser d'homme à nous dans le développement d'un même thème.

S'il est superflu d'examiner les anaphoriques proprement dits, deux exemples méritent cependant d'être cités. Le premier oppose crûment deux antagonistes: un homme typique, le libertin, et "nous", son interlocuteur qui n'est pas dupe de ses fausses libertés : « quel avantage y a-t-il pour nous à ouïr dire à un homme qu'il a donc secoué le joug [...] ? Pense-t-il nous avoir porté par là à avoir désormais bien de la confiance en lui [...] ? Prétendent-ils nous avoir bien réjoui [...] ?» (681, p. 479). Aucun glissement, c'est clair, ne peut s'effectuer de cet homme-là à nous : le libertin restera, au singulier comme au pluriel, un objet d'étonnement et d'horreur, un objet à examiner.

Le deuxième exemple, mettant en scène l'homme générique, amorce au contraire le remplacement d'homme par nous. Le voici : les philosophes « inspiraient des mouvements de grandeur pure et ce n'est pas l'état de l'homme [...]. Il faut des mouvements de bassesse [...] » (17). « II faut », et non, comme attendu, « il lui faut ». Dans le passage de la troisième personne (non-personne) à l'impersonnel strict, on doit voir non pas une perte de personne mais au contraire, paradoxalement, une personnalisation relative. En effet le jeu sur les personnes fait glisser le lecteur d'une attitude d'observation vers une attitude d'implication par suppression du caractère extérieur de son regard. "Il faut" me concerne moi, lecteur, beaucoup plus que ne le ferait « il lui faut ", qui ne toucherait que l'homme en général. Je suis exclu en effet de la troisième personne, mais non d'une forme qui ne spécifie pas la personne.

19 C'est évidemment avec le remplacement d'homme par nous que se rencontrent les cas les plus nets d'implication du lecteur et de l'auteur. « Après avoir entendu toute la nature de l'homme, il faut, pour faire qu'une religion soit vraie, qu'elle ait connu notre nature " (248). Voilà, parmi de nombreux cas ${ }^{9}$, l'exemple de substitution le plus clair parce que réalisé dans l'espace de texte le plus réduit. On ne saurait mieux dire que nous et homme ne sont en fait que deux points de vue ou, plutôt, deux distances par rapport à une seule et même réalité ${ }^{10}$.

20 Cette particularité en matière d'anaphore trouve même sa contre-épreuve. Les Pensées présentent en effet le cas inverse, où homme prend la place de nous : «nous sommes bien certains que nous n'avons pas mal à la tête [...], mais nous ne sommes pas si assurés que nous choisissons le vrai [...]. L'homme est ainsi fait qu'à force de lui dire qu'il est un sot, il le croit » (132).

21 L'examen des textes révèle donc une double pratique de la part de Pascal. Ou éloigner l'homme, en le confirmant dans sa troisième personne, de façon à en faire un objet de regard (fréquemment objet de risée) et un objet de connaissance face au sujet qui enquête, position qu'occupe l'apologiste et qu'il fait partager à son lecteur. Ou, au contraire, rapprocher l'homme du lecteur et de l'auteur jusqu'à opérer leur fusion: l'investigation du sujet qui s'examine lui-même acquiert alors toute sa dimension vitale. La question de l'homme met en jeu, au-delà de la vérité, le souverain-bien et donc la « morale » et le choix de Dieu. En l'occurrence, la chose représentée par l'idée, elle-même représentée par le mot homme, c'est "nous-même ", c'est " notre tout » (681, p. 475). Le remplacement d'homme par ce déictique qu'est le pronom personnel de première personne fait subrepticement glisser le regard du contenu du texte vers celui qui l'écrit et celui qui le lit. Il n'est aucune autre notion des Pensées qui puisse susciter un tel 
déplacement d'un niveau de réalité à un autre. Je est un homme : quel autre mot le verbe copule saurait-il aussi intimement relier au sujet parlant?

Généralisation et implication apparaissent ainsi comme les deux mouvements inverses et réversibles de l'anthropologie pascalienne.

Il existe quelques cas plus complexes. Mais ils ne font que renforcer le système. Examinons en deux. Le grand fragment sur l'imagination propose ainsi un mouvement de va et vient entre homme et nous qu'on peut décrire ainsi. L'analyse des effets de «cette partie dominante dans l'homme » (78, p. 173) se développe d'abord magistralement sur plusieurs pages ${ }^{11}$. Puis soudain, le point de vue se déplace: «nos magistrats ont bien connu ce mystère [...]. Nous ne pouvons pas seulement voir un avocat en soutane [...]» (pp. 176-177); vraiment, l'imagination «semble nous être donnée exprès pour nous induire à une erreur nécessaire ». "De là viennent toutes les disputes des hommes »: trois lignes plus bas, l'analyse a retrouvé enfin la distance qui était précédemment la sienne.

Un jeu substitutif complet est offert par un autre fragment, bref celui-là : «Grandeur de l'homme. Nous avons une si grande idée de l'âme de l'homme que nous ne pouvons souffrir d'en être méprisés et de n'être pas dans l'estime d'une âme. Et toute la félicité des hommes consiste dans cette estime" (30). Nous, après avoir remplacé homme, est lui-même remplacé par les hommes; et l'homme a successivement, en une sorte de syllepse, une valeur générique ("l'âme de l'homme») et une valeur individualisante («en», c'est-à-dire l'âme de tel homme particulier). Sujet qui désire être objet d'estime; objet d'analyse dont est attendue de l'estime sujet de connaissance qui demande à être reconnu: l'homme apparaît apte à occuper simultanément toutes les positions et à brouiller toutes les distances ${ }^{12}$.

Le second type de remplacement en même contexte, non plus par un pronom personnel anaphorique mais par un substantif, revient à exposer le champ synonymique d'homme et donc à mettre en valeur dans chaque paire formée par homme et par son substitut les traits sémantiques qu'ils ont en commun.

Commençons par écarter le cas de membre, auquel on pouvait penser : il n'apparait pas ! Pascal, en effet, n'emploie jamais homme dans ses développements du thème des « membres pensants» (392, etc.). Tout se passe comme si la «morale chrétienne » avait métamorphosé les humains jusque dans leur dénomination pour les intégrer parfaitement dans le corps mystique du Christ ${ }^{13}$.

27 Restent deux substantifs. Deux cas symétriques de restriction de l'extension de la notion et donc d'enrichissement de sa compréhension : l'élévation de l'homme au rang de roi, et son ravalement au niveau du peuple.

28 "L'homme est visiblement fait pour penser [...]. Or à quoi pense le monde ? [...] à danser [...] , à se faire roi, sans penser à ce que c'est qu'être roi, et qu'être homme » (513). En fait, être homme ou être roi, cela revient au même. Par le truchement de tableaux concrets, et non sans une prime de satisfaction narcissique, ce couple permet de penser à la fois la déchéance de l'homme, à travers l'abaissement du roi jusqu'au rang de ses sujets divertis, et la principauté de l'homme, même nostalgique, à travers celle qui subsiste chez le roi. D'une part, le roi n'est qu'un homme, avide d'un divertissement dont la privation est insupportable. «Un roi sans divertissement est un homme plein de misères » (169). La royauté ne fait donc que porter à sa perfection l'illusion d'une voie purement humaine vers le bonheur. Mais elle ne libère pas de l'humanité. Inversement, tout homme est un 
roi. Mais un « roi dépossédé » (149), en exil loin de son royaume natal, déchu de son rang de lumière et d'innocence, chassé d'auprès Dieu.

Roi devient lors l'appellation de l'homme par excellence, sans valeur hyperbolique. Il abandonne en effet, en passant sous l'œil du moraliste de la lumière naturelle à la lumière surnaturelle, son éclat hyperbolique usuel. Le regard théologique, en situant uniformément le discours sur l'homme au niveau de l'hyperbole, tue celle-ci. On tiendra donc, en retour, homme pour une quasi-litote. Roi est alors chargé de manifester pleinement la duplicité de la nature humaine: l'excès de sa misère, exacerbée par la dépossession, aussi bien que sa paradoxale grandeur, qui consiste à ne pouvoir se satisfaire d'un état qu'une simple créature regarderait comme naturel.

Le remplacement d'homme par peuple, qui semble procéder d'une intention opposée, suggère en fait une leçon peu différente. "L'homme est vain ", peut-on lire au premier degré de la recherche de la raison des effets (127). Au troisième degré, peuple se substitue sans crier gare à homme: «il demeure toujours vrai que le peuple est vain ». Faut-il penser que maintenant l'habile doit se distinguer du peuple dont il épouse paroles et attitudes mais non la pensée? Puisqu'il ne partage pas la vanité de ce dernier, s'imposerait donc l'emploi de deux termes différents. Cependant peuple connote à la fois l'ignorance naturelle de l'homme et le bien-fondé de son comportement, conforme aux exigences de la nature déchue ${ }^{14}$. L'homme dans son ignorance essentielle est donc peuple et l'on peut dire qu'à la réalité de la condition humaine (à laquelle l'habile ne peut échapper que spéculativement) convient mieux l'appellation de peuple, plus précise que celle d'homme. Cette restriction en extension s'avère être une clarification en caractérisation. C'est ce dont rend compte l'aptitude de monde à remplacer aussi bien peuple qu'homme: cette fausse spécificité du peuple, l'ignorance, est étendue à la généralité des hommes ${ }^{15}$.

31 Si les substitutions sont instructives (et d'autant plus instructives que discrètes), on peut escompter que, dans une pensée aussi fortement organisée que celle de Pascal en couples de notions antithétiques et en triades, les contrastes seront plus nombreux et plus éclairants. Commençons par les dyades que l'on peut ramener au nombre de sept.

Homme s'oppose d'abord à univers. Ce contraste lui confère "son propre ", à savoir la pensée : «l'homme n'est qu'un roseau, le plus faible de la nature, mais c'est un roseau pensant [...]. Mais quand l'univers l'écraserait, l'homme serait encore plus noble que ce qui le tue ${ }^{16} »(231)$. On ne doit cependant pas oublier que la leçon de cette célèbre métaphore s'expose également en dehors de toute dyade avec l'affirmation bien connue «je ne puis concevoir l'homme sans pensée » (143).

Dans la même optique, l'animalité ne peut être attribuée à l'homme. Les impies se trompent : « ne voyons-nous pas, disent-ils, mourir et vivre les bêtes comme les hommes [...] ? » (183). S'ils ont raison de reprendre, même involontairement, une partie de la leçon divine (" aujourd'hui l'homme est devenu semblable aux bêtes », 182, p. 229), ils ont tort en ce qu'ils en oublient, à savoir la création de l'homme dans un état de perfection. D'ailleurs, dans la pratique, ils se contredisent et distinguent bien homme et animal: " ceux qui méprisent le plus les hommes et les égalent aux bêtes, encore veulent-ils en être admirés et crus » (707). Ils pressentent donc ce qu'ils ignorent : la double nature de l'homme. En effet, «sa nature étant aujourd'hui pareille à celle des animaux », « ce qui est nature aux animaux, nous l'appelons misère en l'homme » (149). 

simple animalité. C'est à l'intérieur même de l'humanité que la maxime lapidaire " poète, et non honnête homme » (503) opère une coupure radicale. Cette condamnation revient plusieurs fois dans les Pensées, telle quelle (613) ou sous forme de variante, poète se remplaçant fort bien, au choix, par auteur (554), par mathématicien ou guerrier (502), par mathématicien ou prédicateur (532). Antithèses instructives. Dans son opposition au spécialiste, enfermé dans les limites de son métier et dont les compétences ajustées à une fraction de l'activité sociale semblent épuiser sa capacité d'humanité (le mathématicien «me prendrait pour une proposition»), l'homme se voit en effet confier le pôle de l'universalité. Il faut « un honnête homme, qui puisse s'accommoder à tous mes besoins généralement " (502). De là découle la belle définition pascalienne de l'honnêteté. « cette qualité universelle » (532), qui se nourrit de la tension entre le particulier et le général: «il est plus beau de savoir quelque chose de tout que de savoir tout d'une chose. Cette universalité est la plus belle » (228).

En revanche dans les contextes que cette opposition n'oriente pas, c'est-à-dire en l'absence du pôle poète, naît une autre opposition, cette fois entre homme et honnête homme. On sait que l'honnêteté ne fait que couvrir le « vilain fond de l'homme » : « il n'est pas ôté » (244). Face à l'honnête homme, bloc d'amour-propre adouci d'un vêtement de civilité, se dresse l'homme chrétien, animé d'une charité intime. On voit donc qu'honnête homme est scindé en deux significations opposées qui occupent tour à tour le pôle positif, dans le couple qu'il forme avec poète, et le pôle négatif, dans celui qui le met en présence de vrai chrétien. Approximation de la charité chrétienne, comme le montrera Nicole dans son essai De la Charité et de l'amour-propre, l'honnêteté n'en est véritablement chez Pascal qu'une image, plaquée sur un cour inchangé et demeuré mauvais. Contre la superficielle honnêteté mondaine est revendiquée une réelle honnêteté de cour. La pensée de derrière, absente de l'opposition entre honnête homme et spécialiste, reprend ici tous ses droits. L'homme se regarde non plus du point de vue de la société civile mais de celui de la Révélation qui enseigne la gravité de la corruption et indique dans le Christ la voie unique de la régénération.

C'est ce que montre bien l'opposition suivante entre homme et saint, recouvrant visiblement celle des deux natures de l'homme. Si la sainteté échappe à l'humanité, c'est par une guérison en quelque sorte miraculeuse qui soigne les effets de la Chute. «Pour faire d'un homme un saint, il faut bien que ce soit la grâce. Et qui en doute ne sait ce que c'est que saint, et qu'homme » (440). La sainteté est ainsi soustraite à la corruption au point de pouvoir être radicalement opposée à l'humanité dominée par sa nature déchue et privée de toute grandeur. Homme devient alors synonyme d'homme sans Dieu. Toute similitude entre ces opposés ( la vie ordinaire des hommes est semblable à celle des saints », 306) ne trouve alors de fondement que figuratif : cherchant les uns et les autres « leur satisfaction », ils ne diffèrent que par l'objet de leur quête.

A ces couples relevant de la nature créée s'ajoutent ceux qui relient et opposent l'homme à la divinité sous les formes de Dieu et de Jésus-Christ. Face à Dieu, ce qui est signifié de l'homme, c'est moins son statut de créature que sa relation de pécheur à celui qui a le projet de le sauver. L'homme est donc dans cette dyade doté d'une histoire beaucoup plus que d'une nature. En classant les fragments concernés, on peut aisément établir un abrégé de cette histoire où se joue le salut d'un homme qui à la fois « est fait » et n'est pas fait « pour Dieu » (18). «L'homme n'est pas digne de Dieu [...]. Il est indigne de Dieu de se joindre à l'homme misérable» (271). Ce Dieu vengeur, qui a chassé l'homme de sa 
proximité et de sa communication, a abandonné la cité terrestre et « pour la punition des hommes les a asservis à ces folies » (48). On ne peut cependant juger si «l'homme est trop peu pour mériter la communication avec Dieu» (263). D'ailleurs, «il y a un devoir réciproque entre Dieu et les hommes" (428). En fait, il nous sauve. "Étant tous corrompus et incapables de Dieu, Dieu s'est fait homme pour s'unir à nous. » (413). En lui donc résident bonheur et vérité : « Misère de l'homme sans Dieu. [...] Félicité de l'homme avec Dieu » (40). Cette vision de Dieu comme sauveur a deux conséquences. D'une part, elle définit bien l'humanité comme pécheresse : le couple Rédempteur/sauvé l'emporte sur le couple Créateur/créature. D'autre part, les figures de Dieu et de Jésus-Christ tendent à se superposer: dans le texte des Pensées, c'est plus souvent Dieu que Jésus-Christ qui sauve l'homme.

La dyade formée par Jésus-Christ et homme présente une organisation très différente. Jésus est en effet à la fois homme et placé face à l'homme. Cette distinction institue deux contextes. L'un, théologique, expose le mystère de l'Incarnation dans lequel la notion d' homme est paradoxalement appliquée à Dieu. L'autre, eschatologique, fait de l'homme le racheté de Jésus-Christ. Passons sur ce dernier contexte, en un sens peu original, et qui surtout ne fait que confirmer ce trait essentiel de l'humanité qui vient d'être souligné ${ }^{17}$. Dans le premier contexte, celui de l'Incarnation, Jésus-Christ n'apparaît pas tellement homme et Dieu mais bien plutôt Dieu caché sous le voile de l'humanité. "Comme Jésus-Christ est demeuré inconnu parmi les hommes, ainsi sa vérité demeure parmi les opinions communes » (258). En revanche celui qui sait voir les grandeurs spirituelles peut s'exclamer «quel homme eut jamais plus d'éclat?» (736). La théologie du «Deus absconditus» exige alors que soit recherché et révélé en quoi Jésus passe infiniment l'homme. L'Incarnation se présente donc surtout comme dépassement de l'humanité. Ainsi, «il n'est pas possible de prendre un homme pour être ressuscité » (353). «Tout homme peut faire ce qu'a fait Mahomet [...]. Nul homme ne peut faire ce qu'a fait Jésus-Christ ${ }^{18}$ " (352). Finalement, "l'Incarnation montre à l'homme la grandeur de sa misère » (384). De même que l'homme ne se réduit pas à son animalité (la similitude entre homme et bête n'est que de seconde nature), Jésus-Christ échappe à son humanité : l'optique des Pensées est ascendante.

39 À ces dyades s'ajoutent ou, mieux, se superposent deux triades qui les combinent partiellement : Dieu, Jésus-Christ et homme; ange, homme et bête. Elles mettent en place une structure commune : le terme médian, homme d'un côté, Jésus-Christ de l'autre, appartient à la fois à deux ensembles, tandis que les termes extrêmes ne relèvent chacun que d'un seul. La première triade couvre l'univers des créatures, composé des mondes spirituel et corporel : entre la bête, corps sans âme, et l'ange, âme sans corps, se situe l'homme, union d'un corps et d'une âme. La seconde triade relie le monde des créatures, réduit à l'homme (seule créature mixte selon la typologie précédente) au monde divin. Jésus-Christ, Dieu fait homme dans le temps humain, n'est en tant que tel (et non en tant que Fils dans la Trinité éternelle) ni Dieu ni homme strictement puisqu'il est pleinement l'un et l'autre à la fois. Il peut donc, si l'on ose dire, faire le Dieu parce qu'il le demeure totalement. L'homme au contraire, qui a été créé corps et âme (cela est indépendant de la Chute), ne peut réussir à faire l'ange. Il n'est et n'a jamais été ange. Il n'est pas un ange fait bête. Entre l'homme et Jésus-Christ, la différence génétique l'emporte sur la similitude structurelle. (557 et 453 barré). Importante définition négative qu'il ne faudrait pas prendre pour une 
définition exclusive. Ne pas être une bête n'implique pas qu'on ne partage rien avec la bête. Deux des dyades examinées ci-dessus éclairent ce fragment. L'homme en effet a en commun avec l'animal les puissants appétits corporels, mais ne partage rien avec l'ange qu'une ancienne proximité de Dieu. Faire l'ange, c'est, hyperboliquement, vouloir atteindre une vie toute spirituelle avec les seules ressources de la nature déchue et sans le secours de la grâce. Ridicule héroïsme stoïcien qui n'a pas les moyens de ses ambitions et ne fait que néantiser la misère. Le saint au contraire dépasse les contraintes de la nature corrompue sans cesser pour autant d'en ressentir les appels.

41 Si cette première triade situe clairement l'homme au milieu de l'échelle des êtres, la notion de milieu n'en demeure pas moins complexe. Il faut ici comprendre que l'homme occupe à la fois ses deux natures et que cette monstruosité fait coexister en lui deux extrêmes. Ils ne se tempèrent pas l'un l'autre. Et gare à qui tente d'exclure l'un au profit de l'autre ! La nature de l'homme se définit donc par une forme : la dualité.

La seconde triade, composée de Dieu, Jésus-Christ et homme, ne confirme pas seulement l'homme dans son statut d'être créé. Elle entraîne surtout, par l'intensification des contrastes, l'affirmation de l'humanité de Jésus. Elle est ici soutenue avec autant de force que sa divinité : « Jésus-Christ est Dieu et homme » $(291,614)$. Mieux, « l'Église a eu autant de peine à montrer que Jésus-Christ était homme [...] qu'à montrer qu'il était Dieu " (338). Inversement, « les Juifs, en éprouvant s'il était Dieu, ont montré qu'il était homme » (337). Sa fonction de médiateur dérive tout naturellement de cette humanité. «Dieu par Jésus-Christ. [...] Jésus-Christ est donc le véritable Dieu des hommes ${ }^{19} »(221)$. Ainsi donc, opposé uniquement à l'homme, Jésus était affirmé comme Dieu ; situé au contraire entre l'homme et Dieu, il affiche sa spécificité : l'Incarnation. On constate une de fois de plus à quel point il n'est de signification que dans un système d'oppositions. "Que dira-t-on qu'est l'homme?» (574). Si Pascal se garde de définir l'homme, il en dit cependant beaucoup de choses! Il reste en effet à caractériser l'homme et donc à étudier les propositions qui le prennent pour sujet. On peut les ramener à deux affirmations simples et générales.

Premièrement, l'homme est un objet connaissable. Précisons bien : l'homme en général et non tel homme dans sa particularité et son histoire. Ce statut d'objet résulte d'autre part de sa position dans le processus de connaissance: face au groupe des examinateurs humains. Clair («Qui voudra connaître à plein la vanité de l'homme n'a qu’à considérer les causes et les effets de l'amour ${ }^{20} », 32$ ) ou obscur ("Sans ces connaissances divines [...], $s$ [i les hommes] connaissaient l'excellence de l'homme, ils en ignorent la corruption ", 240), l'homme n'en demeure pas moins à lui-même un objet de connaissance possible. On peut donc le décrire, comme se proposent de le faire, même schématiquement, des fragments intitulés « Description de l'homme » (113) ou « Condition de l'homme » (58). On peut aussi énoncer des lois à son propos, comme celle-ci : « tous les hommes se haïssent naturellement l'un l'autre » (243).

La vraie religion conforte ce statut. En effet elle est « vénérable parce qu'elle a bien connu l'homme » (46), et sa connaissance est «inséparable » de celle de «la vraie nature de l'homme " (12). Le christianisme [...] affirme donc que l'homme est un objet d'étude stable, universel et intemporel, et donc d'indentification non problématique. Si le moi se dissout en qualités non essentielles, si le cœur n'est accessible en son fond qu'à Dieu, la nature de l'homme au contraire est connaissable dans sa vérité générique. Notre vérité ne réside donc pas dans notre personne mais dans cette humanité que nous partageons avec tous les autres hommes. S'il est vrai que l'histoire de notre salut a une dimension 
individuelle - sinon pourquoi écrire une apologie destinée à la lecture solitaire ? -, le discours apologétique vise, quant à lui, à placer cette histoire dans la compréhension de l'histoire générale du Salut.

Mais la religion ne fait qu'exposer la vérité divine. Ce statut d'objet connaissable qu'elle avance, Dieu le garantit dans son identité et sa permanence. "La création de l'homme » (11) est bien celle d'un être constant. L'expression «la nature de l'homme " revient d'ailleurs fréquemment ${ }^{21}$. Mais il ne faut pas confondre unicité et unification. Cette nature apparaît en effet simple ou double selon que Pascal adopte le point de vue unilatéral des philosophes, qui n'ont connu qu'une moitié de l'homme, ou qu'il laisse parler sa pensée de derrière. La diversité concrète des hommes, notamment celle des métiers, ne doit donc pas faire perdre de vue l'unité essentielle, celle de la création et de la chute, au regard de laquelle la multiplicité n'est qu'accidentelle et superficielle. « Tout est un, tout est divers. Que de natures en celle de l'homme! Que de vacations» (162) : c'est que «la vraie nature étant perdue tout devient sa nature » (16).

Ce caractère antithétique d'un homme unique, voilà notre deuxième affirmation générale. Mais celle-ci est infléchie selon les trois modes majeurs de la pensée pascalienne : le mode géométrique, fondé sur le principe de non-contradiction, le mode dialectique, apte à saisir l'union des contraires, et le mode symbolique, qui découvre leurs rapports figuratifs.

La première perspective est exclusive par principe: elle doit choisir l'une des deux natures de l'homme. Si elle se colore de pessimisme en privilégiant la nature déchue, c'est par prédilection. "Salomon et Job ont le mieux connu et le mieux parlé de la misère de l'homme » (22). Les Pensées ne demeurent pas en reste! Elles proclament en cascade le «vilain fond de l'homme » (244), la «vanité de l'homme» (32) et « des hommes » (228), «la folie des hommes» (448), «la faiblesse de l'homme» $(68,130,648)$, ou encore sa «bassesse » (86). On pourrait poursuivre. Mais l'examen exhaustif des emplois des Pensées oblige à corriger cette lecture trop partiale tant est séduisante la délectation morose de l'infirmité, et donc à tempérer le radicalisme augustinien qu'on veut parfois relever chez Pascal $^{22}$. En effet "grandeur de l'homme ${ }^{23}$ " revient presque aussi fréquemment que " misère » et ses synonymes, auxquels il est souvent associé de façon qu'aucun des deux états de l'homme ne domine. Cependant cette grandeur est généralement tirée d'un renversement de perspective dans l'évaluation de la misère et relève donc davantage d'une optique figurative.

49 La deuxième perspective reconnaît et maintient les deux termes de l'antithèse. Elle se caractérise donc, logiquement, dans le lexique, par le registre métaphorique de la monstruosité et, dans la syntaxe, par l'emploi de « et ». «Quelle chimère est-ce donc que l'homme, quelle nouveauté, quel monstre, quel chaos, quel sujet de contradiction, quel prodige $^{24} »(164$, p. 211$)$. Non pas une fiction mythologique construite par l'imagination, mais une réalité composite et antithétique, comme en témoignent ces nombreux couples d'opposés, que Pascal ose parfois simplement juxtaposer pour aviver la violence de la contrariété : "grandeur, misère " (506); "bassesse et grandeur " (151); grandeur et misère (182) ; « excellence » et "corruption » $(240,648,690,694,708)$. "Cette double capacité » (386), "cette duplicité de l'homme », qui a fait penser à certains «que nous avions deux âmes » (522), s'affichent également dans une série de dédoublements : « deux avènements. Deux états de la nature de l'homme ${ }^{25}$ » (273), cf. 17) ; « la nature de l'homme se considère en deux manières » (160). Il n'est donc pas étonnant de constater qu'il y a " 2 sortes d'hommes en chaque religion ${ }^{26} »(318)$, et que «le christianisme est étrange : il 
ordonne à l'homme de reconnaître qu'il est vil [...], et lui ordonne de vouloir être semblable à Dieu » (383). C'est par cette étrangeté qu'il est la doctrine la " plus propre à l'homme» (386).

50 La troisième perspective n'oppose pas les deux natures de l'homme, mais les découvre l'une dans l'autre, en un renversement du pour au contre. La liasse "Grandeur » est consacrée à ce paradoxe : "la misère se concluant de la grandeur et la grandeur de la misère » (155). Mais un tel exemple reste rare. Le renversement, loin d'être continuel, s'effectue en effet dans un sens privilégié. «La grandeur de l'homme est si visible qu'elle se tire même de sa misère ${ }^{27} »(149)$. Et une telle affirmation n'est généralement pas renversée. La raison en est que ces deux réalités n'appartiennent pas au même plan. En effet l'homme "est misérable puisqu'il l'est. Mais il est bien grand puisqu'il le connaît " (155) : «La grandeur de l'homme est grande en ce qu'il se connaît misérable » (146). On doit conclure qu'il n'existe pas de symétrie rigoureuse entre grandeur et misère, mais renvoi d'un niveau de réalité à un autre : la nature d'une part, la conscience de la nature d'autre part. En somme, la trace de la première nature ne consiste pas en un vestige effectif, mais en un désir nostalgique de cette nature totalement perdue dont ne subsistent que le creux, le sentiment de vide suscité par son absence. S'il demeurait objectivement tant soit peu de cette première nature, une solution stoïcienne de reconquête de soi-même à partir de cette butte-témoin serait parfaitement fondée. C'est une transformation qu'il faut. Seule la grâce peut l'opérer.

51 «Quand j'ai commencé l'étude de l'homme, j'ai vu que [1]es sciences abstraites ne sont pas propres à l'homme [...]. J'ai cru trouver que c'est le vrai étude qui lui est propre [...]. Mais n'est-ce pas que ce n'est pas encore là la science que l'homme doit avoir, et qu'il lui est meilleur de s'ignorer pour être heureux?» (566). Conclusion certes satisfaisante pour le diverti. Mais aussi, par la gradation des effets, pour celui qui cherche à connaitre l'homme par d'autres voies que l'étude : par la voie qu'est Jésus-Christ.

\section{NOTES}

1. L'Esprit géométrique, in CEuvres complètes, éd. J. Mesnard, Bibliothèque européenne, Desclée de Brouwer, t. III, 1991, p. 396.

2. Ibid., p. 397.

3. Voir A Concordance to Pascal's Pensées, Davidson et Dubé, Cornell University Press, Ithaca and London, 1975. Déduction faite des occurrences barrées, de toutes manières généralement non retenues dans l'édition Sellier des Pensées (Classiques Garnier, 1991) qui sera notre édition de référence, homme apparaît 304 fois au singulier et 227 au pluriel (sans différence significative donc entre les deux), chose(s) 380 fois. On peut se contenter, pour dresser le simple classement des substantifs, de l'« Index Words in Order of Frequency », ibid., pp. 1461 et suivantes.

4. 33 raturages (essentiellement dans les fr. 78, 164, 168 et 230) contre 7 dans les unités non classées.

5. On relève en effet homme(s) 126 fois avant la liasse «APR », pour 63 pages de l'édition Sellier, et, à partir d'« APR », 119 fois pour 92 pages : soit une fréquence de 2,1 par page contre 1,3. Le 
mode de calcul est bien sûr grossier ! Mais l'importance de cette différence de fréquence n'en demeure pas moins significative.

6. Grammaire de Port-Royal (chap. VII, Les Articles), Bailly, Hachette, 1846, p. 69 : « le nom commun [...] avec l'article le signifie ou l'espèce dans toute son étendue ; [ou] un ou plusieurs singuliers déterminés par les circonstances de celui qui parle, ou du discours [...]. Avec l'article un [...] [il] signifie un ou plusieurs individus vagues ».

L'abbé Duclos note dans sa Remarque: les noms communs "peuvent être considérés universellement, particulièrement, singulièrement, collectivement ou distributivement. Tout homme marque distributivement l'universalité des hommes: c'est les prendre chacun en particulier. Les hommes marquent l'universalité des hommes: c'est toujours une proposition universelle. Quelques hommes marquent des individus particuliers : c'est le sujet d'une proposition particulière. Le roi, fait le sujet d'une proposition particulière ; le peuple, l'armée [...] sont des collections considérées comme autant d'individus séparés » (ibid., p. 72).

7. Autre exemple : «Les misères de la vie humaine ont frondé tout cela. Comme ils ont vu cela ils ont pris le divertissement » (44).

8. Un autre cas de généralisation se rencontre dans le contexte des citations tirées de l'Ancien Testament, où l'on doit comprendre que les hommes ne désignent que les juifs $(322,324)$. Ce peuple focalise alors l'attention parce que l'histoire du salut se joue chez lui.

9. Voir, entre autres : «sans Jésus-Christ il faut que l'homme soit dans le vice et dans la misère. Avec Jésus-Christ l'homme est exempt de vice et de misère. En lui est toute notre vertu et toute notre félicité » (35). Ou, avec " chacun » pour adoucir la transition : "c'est une chose déplorable de voir tous les hommes ne délibérer que des moyens et point de la fin. Chacun songe [...] mais pour le choix de la condition, de la patrie, le sort nous le donne » (226). Ou, au pluriel : «toutes les occupations des hommes [...]. Nous sommes incapables [...]» (62).

10. Une preuve subsidiaire en est fournie par la facilité qu'il y a à manipuler des énoncés appartenant à une même liasse. En modifiant la modalité mais non la signification strictement anthropologique, on pourrait écrire au choix dans les fragments suivants l'homme, nous ou les hommes: «l'homme ne sait à quel rang se mettre... Il le cherche partout avec inquiétude » (19); "nous souhaitons la vérité et ne trouvons en nous qu'incertitude» (20); "les hommes sont si nécessairement fous...» (31). De même entre les fr. 231 et 232. Un raturage d'homme au profit de nous $\left(164\right.$, p. $\left.86,5^{\circ} \S\right)$ confirme ces remarques.

Exceptionnellement je peut remplir la même fonction: voir le fr. 502. De même on: "cette religion consiste à croire que l'homme est déchu d'un état de gloire [...], mais qu'après cette vie on sera rétabli » (313). On notera que Pascal n' emploie jamais vous dans cette situation de façon à ne pas s'arroger le privilège (ou se donner le ridicule) d'échapper seul à la «condition de l'homme ».

11. Cette imagination "a grand droit de persuader les hommes ", "a établi dans l'homme une seconde nature » (78, p. 174), meut « presque toutes les actions des hommes» (p. 176).

12. Voir également le long fragment «disproportion de l'homme» (230), aux nombreux déplacements de point de vue.

13. Ce que confirme une correction du fr. 309 où hommes est barré au profit de membres.

14. Ainsi, au fr. 116, l'ensemble formé par «tous les hommes» est-il divisé en "peuple», « habiles» et « ceux qui font les entendus ».

15. Voir notamment les fr. 61 («la nature de l'homme n'est pas d'aller toujours [...]. Les inventions des hommes de siècle en siècle [... ]. La bonté et la malice du monde en général [...]») et 513.

16. Voir aussi le fr. 145.

17. Voir par exemple les fr. 35 (cité n. 9) ou 302 («Jésus-Christ n’a fait autre chose qu'apprendre aux hommes qu'ils s'aimaient eux-mêmes [...], qu'il fallait qu'il les délivrât »). 
18. C'est vrai également de ses leçons : «Qu'il enseignerait aux hommes la voie parfaite. Et jamais il n'est venu, ni devant ni après, aucun homme qui ait enseigné rien de divin approchant de cela » (356).

19. Ce fragment, écrit essentiellement à la première personne du pluriel, offre par ailleurs un intéressant exemple de substitution d'hommes à nous.

20. Voir aussi le fr. 160.

21. 11 fois, plus 3 fois « la nature des hommes » $(680,684,708)$. De même, 4 fois « la condition de l'homme » contre 1 fois « la condition des hommes » (686). La prédominance du singulier signale une fois de plus la vision généralisante de Pascal.

22. On notera par exemple que la vision de l'homme qui se déduit des exemples du Dictionnaire universel de Furetière rejoint beaucoup plus dans son équilibre celle de Pascal que celle, très pessimiste comme on va le voir immédiatement, du Dictionnaire de l'Académie : « la condition de l'homme est bien malheureuse. L'homme est sujet à beaucoup d'infirmités. Tous les hommes sont sujets à la mort. Tous les hommes ont péché en Adam. Tous les hommes ont été rachetés par Jésus-Christ [...]».

23. Bien sûr dans toute la liasse "Grandeur ", mais aussi dans les fr. 30, 151, 628, 648; 153, 690 (« sa grandeur »); 182 (« grandeur en l'homme »); etc.

24. Voir aussi les fr. 163, 240 et 712.

25. Voir aussi les fr. 17 et 291 (« deux avènements, l'un de misère pour abaisser l'homme superbe, l'autre de gloire pour élever l'homme humilié »).

26. Voir aussi les fr. 209 et 398.

27. Voir aussi les fr. 148 et 150 («grandeur de l'homme dans sa concupiscence même »).

INDEX

Mots-clés : Pascal, Pensées, homme

Keywords : human

\section{AUTEUR}

\section{CHRISTIAN MEURILLON}

Maître de conférences

Université Lille 3 